\title{
Electrically controlled phases of partially polarized light and orientational Kerr effect in liquid crystal ferroelectrics
}

\author{
Alexei D. Kiselev ${ }^{1, \star}$, Vladimir V. Kesaev ${ }^{2, \star \star}$, and Evgeny P. Pozhidaev ${ }^{2, \star \star \star}$ \\ ${ }^{1}$ Saint-Petersburg National Research University of Information Technologies, Mechanics and Optics, 49 \\ Kronverksky Pr., St. Petersburg 197101, Russia \\ ${ }^{2}$ Lebedev Physical Institute RAS, 53 Leninskiy Ave., Moscow 119991, Russia
}

\begin{abstract}
We study the electro-optic properties of subwavelength-pitch deformed-helix ferroelectric liquid crystals illuminated with partially polarized light. In an experimental setup based on the Mach-Zehnder interferometer, it is found that the interference pattern crucially depends on the degree of polarization of the incident light. We evaluate the electric field dependence of both the Pancharatnam relative phase and the geometric phase for the general case of nonunitarily evolving mixed polarization states.
\end{abstract}

Liquid crystal (LC) spatial light modulators have been widely employed to modulate amplitude, phase, or polarization of light waves in space and time. Ferroelectric liquid crystals (FLCs) represent most promising chiral liquid crystal material which is characterized by very fast response time. However, most of the FLC modes are not suitable for phase-only modulation devices because their optical axis sweeps in the plane of the cell substrate producing undesirable changes in the polarization state of the incident light. In order to get around the optical axis switching problem the system based on the orientational Kerr effect in a vertically aligned deformed helix ferroelectric LC (DHFLC) with subwavelength helix pitch was suggested in $[1,2]$. In the geometry of a uniform lying helix, phase modulation of light in DHFLC cells was also studied using the experimental technique based on a Mach-Zehnder two-arm interferometer [3].

In our recent paper [4], we have found that, for unpolarized incident light, the electro-optic response is insensitive to the effect of electric-field-induced rotation of in-plane optical axes and can be described in terms of the electrically dependent Pancharatnam phase. In this paper, we examine the electro-optic behavior of the planar aligned DHFLC cells illuminated with partially polarized light and extend our theoretical analysis of the Pancharatnam and geometric phases to the general case of mixed polarization states that undergo nonunitary and non-cyclic evolution.

We consider a DHFLC layer of thickness $D$ with the $z$ axis normal to the bounding surfaces. The geometry of a uniform lying DHFLC helix with subwavelength pitch where the helix (twisting) axis $\hat{\mathbf{h}}$ is directed along the $x$ axis will be our primary concern. According to [3, 5, 6], optical properties of such cells can be described by the effective dielectric tensor of a homogenized DHFLC helical structure. The electric-field-induced anisotropy is generally biaxial so that the dielectric tensor is characterized by the three generally different principal values (eigenvalues): $\epsilon_{ \pm}=n_{ \pm}^{2}$ and $\epsilon_{z}=n_{z}^{2}$.

\footnotetext{
$\star$ e-mail: alexei.d.kiselev@gmail.com

$\star \star$ e-mail: vladimir.kesaev@gmail.com

$\star \star \star$ e-mail: epozhidaev@mail.ru
} 
The in-plane principal optical axes (eigenvectors) $\hat{\mathbf{d}}_{+}=\cos \psi_{d} \hat{\mathbf{x}}+\sin \psi_{d} \hat{\mathbf{y}}, \quad \hat{\mathbf{d}}_{-}=\hat{\mathbf{z}} \times \hat{\mathbf{d}}_{+}$are rotated about the vector of electric field, $\mathbf{E} \| \hat{\mathbf{z}}$, by the azimuthal angle $\psi_{\mathrm{d}}$. In the low electric field region, the electric field dependence of the angle $\psi_{\mathrm{d}}$ is approximately linear: $\psi_{\mathrm{d}} \propto E$, whereas the electrically induced part of the principal refractive indices, $n_{ \pm}$and $n_{z}$, is typically dominated by the Kerr-like nonlinear terms proportional to $\left.E^{2}[3,6]\right)$.

The input lightwave field is characterized by the polarization density matrix $\rho_{0}$ that, in the circular basis $\hat{\mathbf{e}}_{ \pm}=(\hat{\mathbf{x}} \pm i \hat{\mathbf{y}}) / \sqrt{2}$, can be written in the form: $2 \rho_{0}=\sigma_{0}+P_{0}\left(\hat{\mathbf{s}}_{0} \cdot \boldsymbol{\sigma}\right), \sigma_{0}=\operatorname{diag}(1,1), \boldsymbol{\sigma}=$ $\left(\sigma_{1}, \sigma_{2}, \sigma_{3}\right)$, where $\sigma_{i}$ are the Pauli matrices; $\hat{\mathbf{s}}_{0}=\left(\sin \left(2 \theta_{0}\right) \cos \left(2 \phi_{0}\right), \sin \left(2 \theta_{0}\right) \sin \left(2 \phi_{0}\right), \cos \left(2 \theta_{0}\right)\right)$ is the normalized Stokes vector and $P_{0}$ is the degree of polarization. Similarly, the transmission matrix of the DHFLC cell, $\mathbf{T}_{s}(h)$, where $h=k_{\mathrm{vac}} D=\omega / c D$ is the thickness parameter, can be expressed in terms of the vector $\hat{\mathbf{s}}_{d}=\left(\cos 2 \psi_{d}\right.$, $\left.\sin 2 \psi_{d}, 0\right)$ that can be regarded the normalized Stokes vector of a light beam linearly polarized along the optic axis $\hat{\mathbf{d}}_{+}$and the transmission coefficients, $t_{+}$and $t_{-}[6]$.

We can now apply the interferometry based approach formulated in [7] to the case of plane wave which is normally incident on the DHFLC cell and is characterized by the polarization density matrix $r h o_{0}$ and derive the expressions for the Pancharatnam and geometric phases, $\Phi_{P}$ and $\Phi_{g}$, acquired by the light transmitted through the DHFLC cell. The result reads

$$
\begin{aligned}
& \Phi_{P}=\Phi+\arg \left[\operatorname{Re} \tilde{F}_{P}+i \tilde{P} \operatorname{Im} \tilde{F}_{P}\right]=\arg \operatorname{Tr}\left[\mathbf{T}_{s}(h) \rho_{0}\right] \\
& \tilde{F}_{P}=\cos \Delta \Phi \cos \left[\theta_{\lambda}(h)-\theta_{\lambda}(0)\right]+i \sin \Delta \Phi \cos \left[\theta_{\lambda}(h)+\theta_{\lambda}(0)\right], \\
& \Phi_{g}=\arg \left[\operatorname{Re} \tilde{F}_{g}+i \tilde{P} \operatorname{Im} \tilde{F}_{g}\right], \tilde{F}_{g}=\tilde{F}_{P} \mathrm{e}^{-i \tilde{\Phi}_{d}}, \\
& \tilde{\Phi}_{d}=\int_{0}^{h} \cos \left(2 \theta_{\lambda}(s)\right) \Delta \Phi_{s}^{\prime}(s) \mathrm{d} s, \cos \left(2 \theta_{\lambda}\right)=q_{3} /|\mathbf{q}|,|\mathbf{q}|=\sqrt{q_{3}^{2}+q_{0}^{2}\left[1-\left(\hat{\mathbf{s}}_{d} \cdot \hat{\mathbf{s}}_{0}\right)^{2}\right]}
\end{aligned}
$$

where $\Phi=\left(\Phi_{+}+\Phi_{-}\right) / 2$ is the averaged phase shift; $\Delta \Phi=\left(\Phi_{+}-\Phi_{-}\right) / 2$ is the phase retardation; $\tilde{P}=\frac{\sqrt{p_{+}(0) p_{+}(h)}-\sqrt{p_{-}(0) p_{-}(h)}}{\sqrt{p_{+}(0) p_{+}(h)}+\sqrt{p_{-}(0) p_{-}(h)}}, p_{\mu}(0)=\frac{1+\mu P_{0}}{2}, p_{\mu}(h)=\frac{\operatorname{Tr}(\rho)+\mu|\mathbf{q}|}{2}, q_{0}=P_{0}\left[\tau_{+}^{2}-\tau_{-}^{2}\right]$, $q_{3}=2 \tau_{-} \tau_{+}+P_{0}\left[\tau_{+}^{2}+\tau_{-}^{2}\right]\left(\hat{\mathbf{s}}_{d} \cdot \hat{\mathbf{s}}_{0}\right), \operatorname{Tr}(\rho)=\tau_{+}^{2}+\tau_{-}^{2}+2 P_{0} \tau_{+} \tau_{-}\left(\hat{\mathbf{s}}_{d} \cdot \hat{\mathbf{s}}_{0}\right), \tau_{ \pm}=\left(\left|t_{+}\right| \pm\left|t_{-}\right|\right) / 2$.

In conclusion, our analytical results show that, in contrast to the total Pancharatnam phase, the geometric phase is determined by the phase retardation and is independent of the averaged phase shift. In addition, the electric field dependence of the phases enters through the angle between the Stokes vectors $\hat{\mathbf{s}}_{0}$ and $\hat{\mathbf{s}}_{d}$ decribing the effect of electrically induced rotation of the optical axes. This effect is supressed in the limiting cases of unpolarized and circular polarized incident light. The effects of nonunitarity are governed by the difference between the transmittance coefficients: $\left|t_{+}\right|-\left|t_{-}\right|$.

We acknowledge financial support from RFBR under Grants 16-02-00441 a, 16-29-14012 ofi_m and 16-42630773 p_a.

\section{References}

[1] E.P. Pozhidaev, A.D. Kiselev, A.K. Srivastava, V.G. Chigrinov, H.S. Kwok, M.V. Minchenko, Phys. Rev. E 87, 052502 (2013)

[2] E.P. Pozhidaev, A.K. Srivastava, A.D. Kiselev, V.G. Chigrinov, V.V. Vashchenko, A.V. Krivoshey, M.V. Minchenko, H.S. Kwok, Optics Letters 39, 2900 (2014)

[3] S.P. Kotova, S.A. Samagin, E.P. Pozhidaev, A.D. Kiselev, Phys. Rev. E 92, 062502 (2015)

[4] V.V. Kesaev, A.D. Kiselev, E.P. Pozhidaev, Phys. Rev. E 95, 032705 (2017)

[5] A.D. Kiselev, E.P. Pozhidaev, V.G. Chigrinov, H.S. Kwok, Phys. Rev. E 83, 031703 (2011)

[6] A.D. Kiselev, V.G. Chigrinov, Phys. Rev. E 90, 042504 (2014)

[7] D.M. Tong, E. Sjöqvist, L.C. Kwek, C.H. Oh, Phys. Rev. Lett. 93, 080405 (2004) 